\title{
Static Friction Detection Based on Artificial Neural Networks Method
}

\section{Detección de fricción estática basado en el método de redes}

neuronales artificiales

Author:

Muhammadamin Daneshwar

Sirwan Mohamad Kekshar ${ }^{2}$

Sadegh Aminifar ${ }^{3}$

\section{SCIENTIFIC RESEARCH}

How to cite this paper:

How to cite this paper: Daneshwar. M., Kekshar S. M., Sadegh. A. Static Friction Detection Based on Artificial Neural Networks Method.Innovaciencia 2019; 7(1): $1-7$.

http://dx.doi.org/10.15649/2346075X.518

Reception date:

Received: 19 June 2019

Accepted: 20 September 2019

Published: 25 October 2019

Keywords:

static friction, model, static friction, neural networks.

\begin{abstract}
Introduction: Poor product quality and high energy consumption of many control loops is due to the presence of static friction. This phenomenon is monitored by human in many industrials. The decision is made based on human's brain which is not effective and reliable. Methods: A model-based method of stiction detection based on an artificial neural network (ANN) is proposed. The ANN which is run in parallel to the process predicts a dynamic model of the process using data obtained from control signal and process output. Results: It can be seen that the proposed method based on ANN can be replaced with human monitoring method. Conclusions: Capability of the proposed method of static friction detection for the process with the sticky valve is confirmed by data obtained from the simulation in a control loop with sticky valve.
\end{abstract}

1 Muhammadamin Daneshwar is with the Computer Science Department, Faculty of Science, Soran University, mdaneshwar@ gmail.com.

2 Sirwan Mohamad Kekshar is with the Computer Science Department, Faculty of Science, Soran University, mershefa@gmail. com.

3 Sadegh Aminifar is with the Computer Science Department, Faculty of Science, Soran University, saminifar@yahoo.com. 


\section{INTRODUCCTION}

It has been shown in the literature that, the presence of static friction in the valve is a major factor in oscillations and therefore performance degradation in various control tasks $\frac{(1),(2),(3)}{1}$. The degradation is done by changing the operating conditions from their ideal values. Finally, the valve satiation will lower the product quality and increases the energy consumption. This issue must be monitored continuously; otherwise, its effect can be propagated to entire plant. Therefore many researchers have conducted researches on the presence of static friction as well as dealing with nonlinear and uncertain phenomena $(4),(5),(6)$.

Wide applications of Artificial Neural Networks (ANNs) have attracted the attention of many researchers $(7),(8),(2),(10),(11)$. They are very effective and useful tools in many fields including modeling and identification. The ability to learn and generalize is a special factor for ANNs. They can make a decision like a human. The prediction behavior of nonlinear systems is another useful property of an ANN. In this study by taking advantage of ANN and combining them with other methods, a new method for solving a control valve with stiction is presented.

The remainder of this paper is organized as follows: Some related works have been presented in section 2 . The definition of stiction and therefore the presence of the problem in the control loop is introduced in Section 3. In Section 4, a method to handle and solve the problem is proposed. In Section 5, simulation results are given confirming the performance of the proposed method.

\section{MATERIALS AND METHOD}

\section{Related works}

The available methods of diagnosis of stiction for control loops in the literature suffer from some problems ${ }^{(1)}$. The applications of shape based techniques to flow control loops are limited. This is because of this fact that the flow loop has dynamics that can distort the shape of the stiction pattern. Furthermore, other techniques of stiction detection are not lack of drawback. Some of these techniques and their limitations are: Bicoherence and Ellipse-fitting method of Choudhury ${ }^{(12)}$ which needs significant data points. For clustering based method of Daneshwar ${ }^{(3)}$, also high enough length of data is needed to be clustered. It means that with available methods of clustering, in case of low length of data, the data cannot be

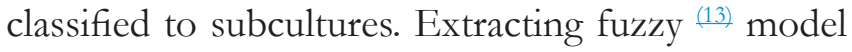
of a process with control valve stiction in ${ }^{(2)}$ needs complex mathematic calculation. A good survey on effective methods of detection and compensation of stiction can be found in $\frac{(14)}{}$.

\section{Definition of Stiction (problem statement)}

Figure 1 shows a typical closed loop control system which suffers from static friction. As it can be seen from the figure, a sticky valve which controls the process can affect the entire control loop.

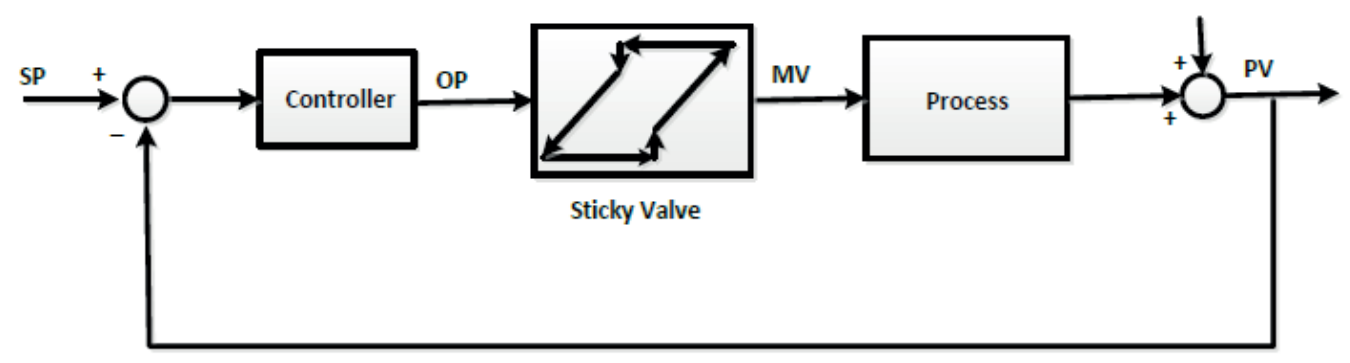

Figure 1. A typical closed loop control system with sticky valve. 
Static friction can be defined based on resistance of a surface against motion of an object in the start of motion. Both data-driven and physical-based approaches are common in the literature to model this undesirable phenomenon. Because the data-driven approach is superior to physical based method, it is considered in this paper. This selection can be prov- en when one can see the application of data-driven in many fields including artificial neural networks, data mining and system identification. Typical input-output behavior of a sticky valve based on choudhury's (12) definition has been shown in Figure 2. It includes stick band (S), slip jumps (J) and moving phase.

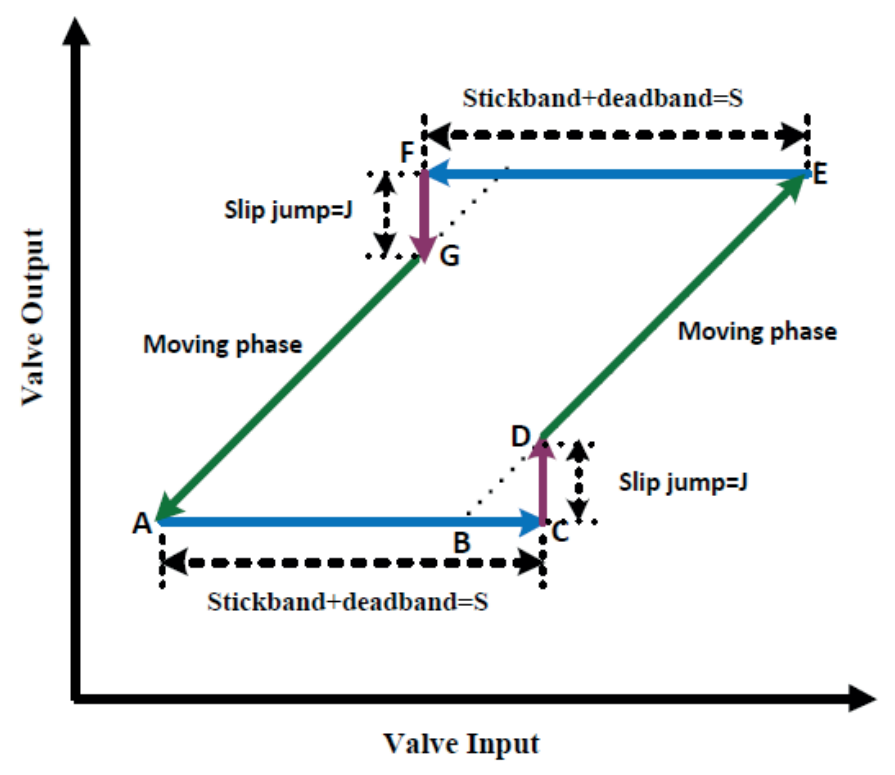

Figure 2. Typical input-output behavior of a sticky valve based on choudhury'sdefinition ${ }^{(12)}$.

Solving the problem using a method base on

\section{Artificial Neural Networks}

Proposed method is based on combining ANN with linear element to predict behavior of a control loop. The loop has a nonlinear element (due to presence of stiction) and linear part which covers the process.
According to figure 3, a similar input is applied to both original system and the model. Due to presence of nonlinear element, MV (output of the valve) is nonlinear. Therefore the final output of the process (PV) is nonlinear. An unknown noise $(\mathrm{E}(\mathrm{t}))$ also can distort the final part of the control system(y). The model consists of ANN and linear element.

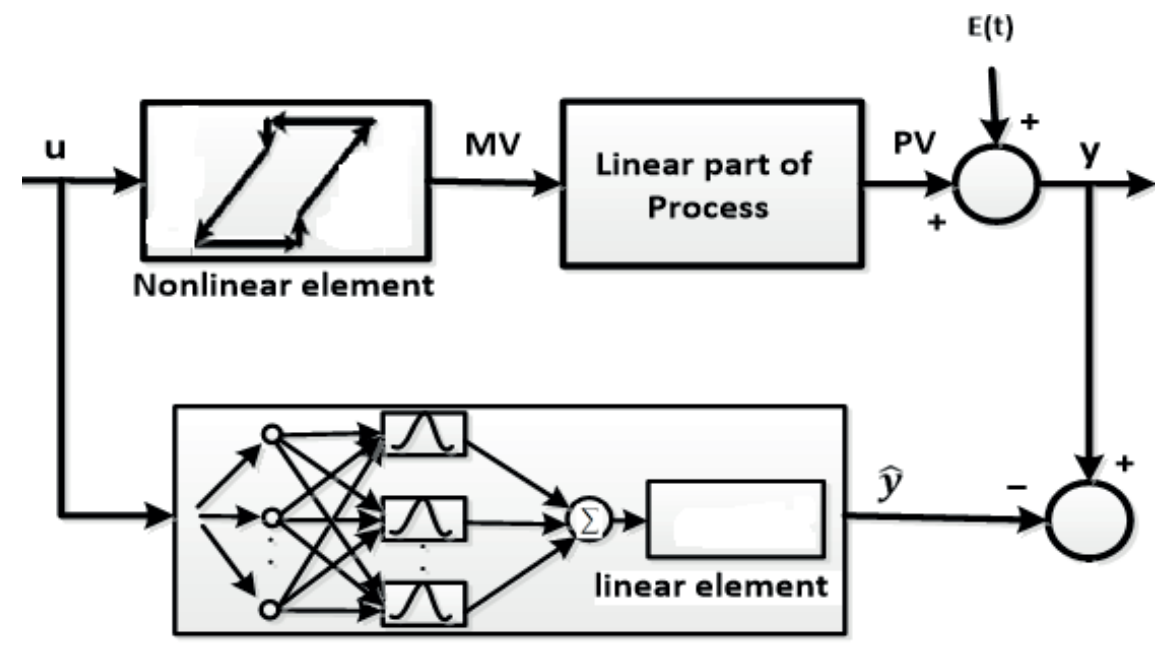

Figure 3. Proposed method for static friction detection in control loops based on ANN. 
For nonlinear part, a three-layer Radial Basis Function (RBF) neural network has been used $\stackrel{(15)}{\text {. }}$ This type of ANN has a three layer: an input layer, a nonlinear hidden layer $(\mathrm{RBF})$ and a linear output layer as shown in Figure 4.

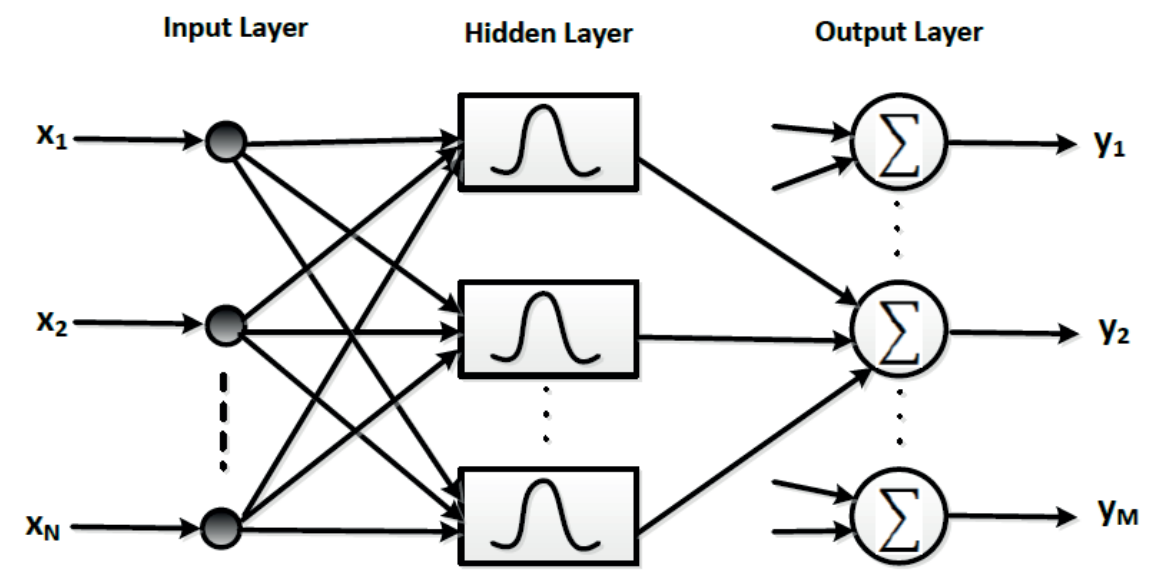

Figure 4. Structure of a radial basis function (RBF) neural network.

The input vector $X=\left[x_{1}, x_{1}, \ldots, x_{N}\right]^{T}$ can be weighted in hidden layer. The hidden layer has a nonlinear function. The final output is obtained when the output $y=\left[y_{1}, y_{1}, \ldots, y_{M}\right]^{T}$ of nonlinear functions (equation 1) are added together. The nonlinear function has a Gaussian shape as defined by the following equation:

$\phi_{i}(X)=\exp \left(-\frac{\left\|x-\mu_{i}\right\|^{2}}{2 \sigma_{i}^{2}}\right)$

Where $\mu_{i}$ and $\sigma_{i}$ denote the center and spread width of the $\mathrm{i}$-th node, respectively.

The network output can be obtained by

$y=f(X)=\sum_{i=1}^{k} w_{i} \phi_{i}(X)$

The linear element of figure 3 is represented by ARMAX model $\stackrel{(16)}{\text {. }}$

$A\left(q^{-1}\right) y(k)=B\left(q^{-1}\right) u(k)+C\left(q^{-1}\right) e(k)$

In the above equation, the backward shift operator is represented by $q^{-1}$, and $\varepsilon$ is the unmeasured disturbance. $\mathrm{A}\left(q^{-1}\right), \mathrm{B}\left(q^{-1}\right)$ and $\mathrm{C}\left(q^{-1}\right)$ are polynomials of specified order $n, m$ and $p$, respectively.

\section{SIMULATION AND RESULTS}

For simulation and therefore confirmation of proposed method, several pairs of data (181 pairs) in a control loops (Figure 1) with static friction have been generated. The obtained data have been used according to figure 3. Data have been classified to set of training and testing of data. 75 percent of data has been used for training of ANN and 25 percent of data has been used for testing of obtained ANN. Overlapping of targets and outputs in both figure 5 and figure 6 shows that the proposed method is able to capture true characteristics of the system with the presence of static friction. The regression in both figure is nearly its perfect value (one) which confirms the performance of the system. Both Mean Squared Error (MSE) and Root Mean Square Error (RMSE) in both figures are in acceptable range (lower than 0.1). Finally, normal distribution of error in last part of both Figure 5 and Figure 6 are another proof for performance of the proposed method. 

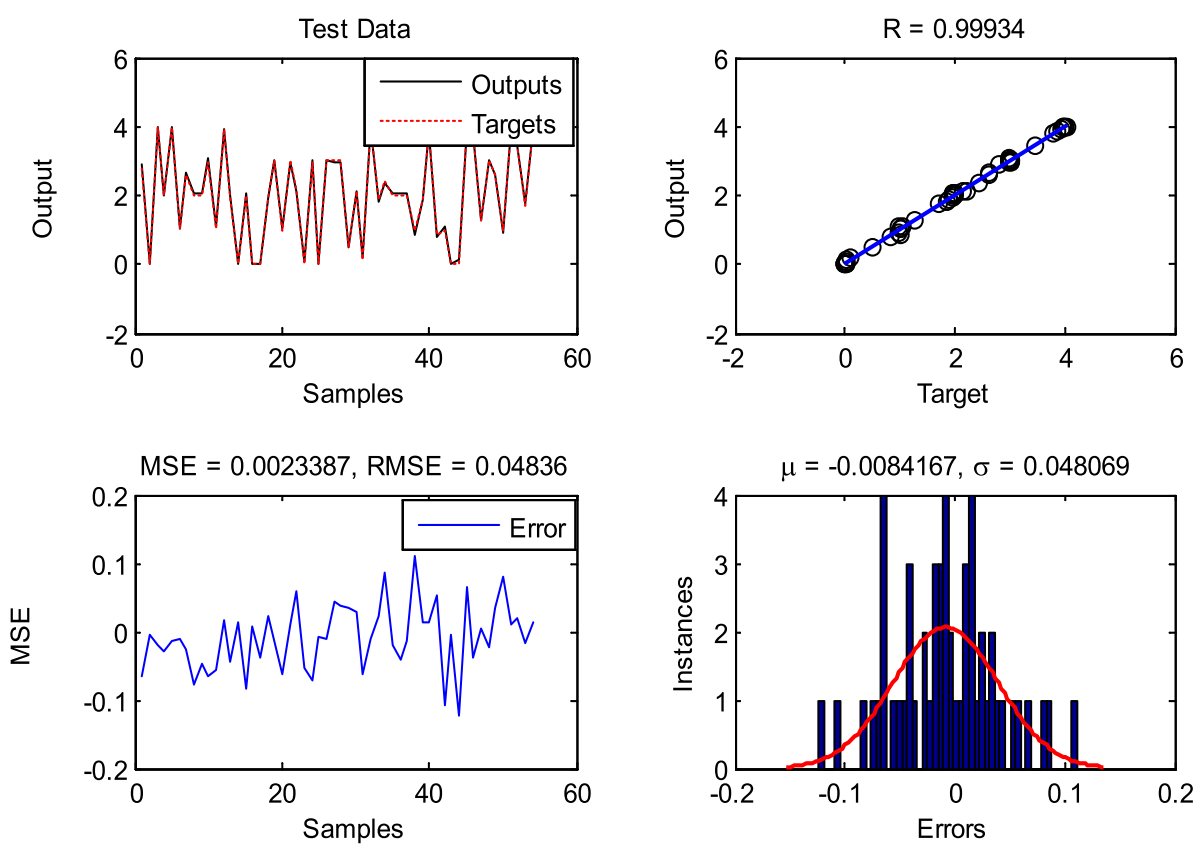

Figure 5. Performance of proposed method based on ANN model on sets of testing data.
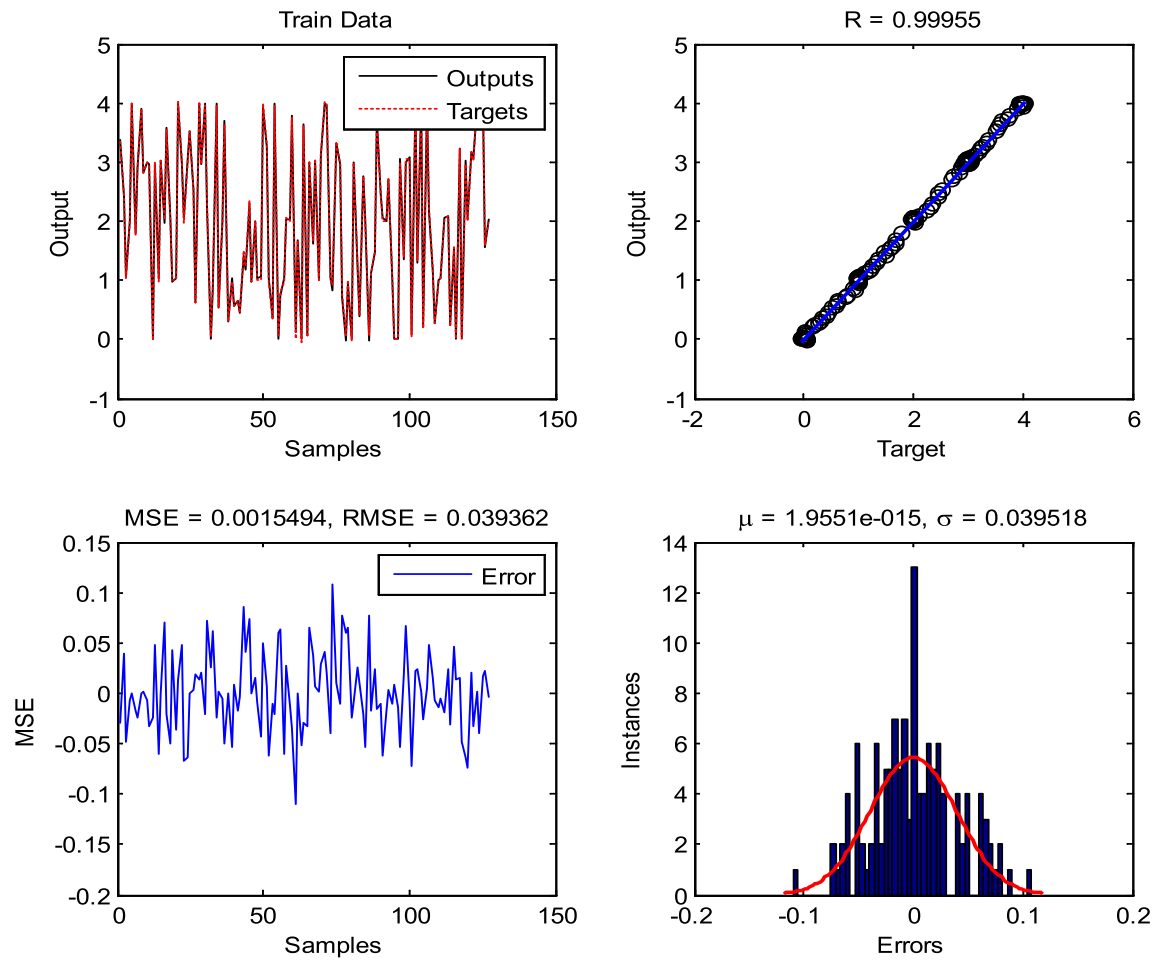

Figure 6. Performance of proposed method based on ANN model on sets of training data.

For evaluating the performance of current work, a comparison of static and proposed ANN based method have been collected in table 1. For the comparison, a control loop with different scenarios has been used. Loop 1 represents weak stiction. Over shoot case has been labeled with Loop 2. In loop 3, strong stiction has been studied. In loop 4, a strong noise has been added to a loop with stiction. The percentage of correct detection has been reached to $75 \%$. 
Table 1. Comparison of static and proposed ANN based method on some loops with different scenarios.

\begin{tabular}{lccccc}
\hline Reference & Loop 1 & Loop 2 & Loop 3 & Loop 4 & $\begin{array}{c}\text { Percentage of } \\
\text { correct detection }\end{array}$ \\
\hline Yamashita, 2006 & Yes & No & No & No & 25 \\
Daneshwar, 2014 & Yes & Yes & No & No & 50 \\
Daneshwar, 2015 & Yes & No & Yes & No & 50 \\
Proposed work & Yes & Yes & No & Yes & 75 \\
\hline
\end{tabular}

\section{CONCLUSIONS AND FUTURE WORK}

As the presence of static friction in control loops degrades its performance, it must be monitored continuously. Human based monitoring system is not reliable and effective. In this paper, a method which combines ANN with a linear element was proposed. Simulation results show that the proposed method which learns from the previous data is able to predict the behavior of a control system with the sticky valve. The proposed method can be used in future compensations methods to mitigate static friction impact on control system.

\section{REFERENCES}

1. Yamashita Y. An automatic method for detection of valve stiction in process control loops. Control Engineering Practice. 2006; 14(5), 503-510.

https://doi.org/10.1016/j.conengprac.2005.03.004.

2. Daneshwar M. A., Noh N. M. Identification of a process with control valve stiction using a fuzzy system: a data-driven approach", Journal of Process Control. 2014; 24, 249-260.

https://doi.org/10.1016/j.jprocont.2014.01.013

3. Daneshwar M. A., Noh N. M. Detection of stiction in flow control loops based on fuzzy clustering", Control Engineering Practice. 2015; 39, pp. 23-34.

https://doi.org/10.1016/i.conengprac.2015.02.002

4. Aminifar S., bin Marzuki A. Horizontal and vertical rule bases method in fuzzy controllers. Mathematical Problems in Engineering, 2013. http://dx.doi.org/10.1155/2013/532046.
5. Aminifar S., Marzuki A. Uncertainty in interval type-2 fuzzy systems. Mathematical Problems in Engineering, 2013. http://dx.doi.org/10.1155/2013/452780.

6. Marzuki A., Tee S.Y., Aminifar S. Study of fuzzy systems with Sugeno and Mamdanitype fuzzy inference systems for determination of heartbeat cases on Electrocardiogram (ECG) signals", International Journal of Biomedical Engineering and Technology. 2014; (3), 243-276. https://doi.org/10.1504/IJBET.2014.059673

7. Maghdid H. S. and Lami I. A. Dynamic clock-model of Wi-Fi access-points to help indoors localisation of smartphones," 2012 IV International Congress on Ultra Modern Telecommunications and Control Systems, St. Petersburg, 2012, pp. 268-273. doi: 10.1109/ICUMT.2012.6459677. https://doi.org/10.1109/ICUMT.2012.6459677

8. Lami I. A. and Maghdid H. S. SynchronisingWiFi access points with GPS time obtained from smartphones to aid localization. 2013 International Conference on Computer Applications Technology (ICCAT), Sousse, 2013, pp. 1-6. doi: 10.1109/ICCAT.2013.6522052.

https://doi.org/10.1109/ICCAT.2013.6522052

9. Sabir A., Ahmed M., Faeq A., and Maghdid H. Human gait identification using Kinect sensor", Kurdistan Journal of Applied Research. 2017; 2(3), 142-146.

https://doi.org/10.24017/science.2017.3.37

10. Maghdid S. A., Maghdid H. S., HmaSalah S. R., Ghafoor K. Z., Sadiq A. S., Khan S. Indoor human tracking mechanism using integrated onboard smartphones Wi-Fi device and inertial sensors. Telecommunication Systems. 2018; pp.1-12. https://doi.org/10.1007/s11235-018-0517-2. 
11. Maghdid H. S. Web News Mining Using New Features: A Comparative Study. IEEE Access. 2019; 7, 1-16. doi: 10.1109/ACCESS.2018.2890088. https://doi.org/10.1109/ACCESS.2018.2890088

12. Choudhury M.A.A.S., Jain M., Shah S. L.Stiction definition, modelling, detection and quantification". Journal of Process Control.2008; 18(3-4), 232-243.

https://doi.org/10.1016/j.jprocont.2007.07.004.

13. YosefiG., AminifarS., NedaS., DaneshwarM.A. Design of a mixed-signal digital CMOS fuzzy logic controller (FLC) chip using new current mode circuits"AEU-International Journal of Electronics and Communications.2011; 65 (3), 173-181. https://doi.org/10.1016/j.aeue.2010.01.019
14. Daneshwar M. A, Noh N. M. Valve stiction in control loops-A survey on effective methods of detection and compensation"2012 IEEE International Conference on Control System, Computing and Engineering. 2012, pp.155-159.

https://doi.org/10.1109/ICCSCE.2012.6487133

15. Tavoosi J., Jokandan A. S., Daneshwar M. A. A new method for position control of a 2-DOF robot arm using neuro-fuzzy controller, Indian Journal of Science and Technology. 2012; 5 (3), 2253-2257.

16. Ljung L. System identification: theory for the user. Prentice Hall PTR, 1999. 